\title{
Amelioration of emphysema in mice through lentiviral transduction of long-lived pulmonary alveolar macrophages
}

\author{
Andrew A. Wilson, ${ }^{1,2}$ George J. Murphy, ${ }^{2,3}$ Hiroshi Hamakawa, ${ }^{4}$ \\ Letty W. Kwok, ${ }^{1,2}$ Sreedevi Srinivasan, ${ }^{5}$ Avi-Hai Hovav, ${ }^{6}$ \\ Richard C. Mulligan, ${ }^{7}$ Salomon Amar, ${ }^{5}$ Bela Suki, ${ }^{4}$ and Darrell N. Kotton ${ }^{1,2}$

\begin{abstract}
1 Pulmonary Center and Department of Medicine, Boston University School of Medicine, Boston, Massachusetts, USA. ${ }^{2}$ Boston University Center for Regenerative Medicine (CReM), Boston, Massachusetts, USA. ${ }^{3}$ Section of Hematology-Oncology and Department of Medicine, Boston University School of Medicine, Boston, Massachusetts, USA. ${ }^{2}$ Department of Biomedical Engineering, Boston University, Boston, Massachusetts, USA. ${ }^{5}$ Department of Periodontology and Oral Biology, Boston University School of Dental Medicine, Boston, Massachusetts, USA. ${ }^{6}$ The Hebrew University Faculty of Dental Medicine, Institute of Dental Sciences, Jerusalem, Israel. ${ }^{7}$ Department of Genetics, Harvard Medical School, Boston, Massachusetts, USA.
\end{abstract}

\begin{abstract}
Directed gene transfer into specific cell lineages in vivo is an attractive approach for both modulating gene expression and correcting inherited mutations such as emphysema caused by human $\alpha 1$ antitrypsin (hAAT) deficiency. However, somatic tissues are mainly comprised of heterogeneous, differentiated cell lineages that can be short lived and difficult to specifically transfect. Here, we describe an intratracheally instilled lentiviral system able to deliver genes selectively to as many as $\mathbf{7 0} \%$ of alveolar macrophages (AMs) in the mouse lung. Following a single in vivo lentiviral transduction, genetically tagged AMs persisted in lung alveoli and expressed transferred genes for the lifetime of the adult mouse. A prolonged macrophage lifespan, rather than precursor cell proliferation, accounted for the surprisingly sustained presence of transduced AMs. We utilized this long-lived population to achieve localized secretion of therapeutic levels of hAAT protein in lung epithelial lining fluid. In an established mouse model of emphysema, lentivirally delivered hAAT ameliorated the progression of emphysema, as evidenced by attenuation of increased lung compliance and alveolar size. After 24 weeks of sustained gene expression, no humoral or cellular immune responses to hAAT protein were detected. Our results challenge the dogma that AMs are short lived and suggest that these differentiated cells may be a possible target cell population for in vivo gene therapy applications, including the sustained correction of hAAT deficiency.
\end{abstract}

\section{Introduction}

Gene transfer into specific cell lineages in vivo remains an attractive yet elusive approach for modulating gene expression or correcting inherited mutations. Although a variety of techniques have been developed to deliver DNA molecules to cells in vitro, in vivo gene transfer has been limited in many cell types by inefficient gene delivery. In addition, selective delivery of genes to subtypes of cells in complex tissues has been challenging. In most cases where target cells have been transduced in vivo, the duration of gene expression has been frustratingly short due to several mechanisms including the limited lifespan of differentiated cell types, the development of cellular or humoral immunity against the vector or transgene, and transgene promoter silencing (1-4).

One approach to surmounting these hurdles is the genomic integration (transduction) of transferred genes into tissue stem cells because stem cells self-renew indefinitely and give rise to nearly unlimited numbers of differentiated progeny throughout the life of an animal (5). An alternative approach is the transduction of differentiated cells. However, some differentiated cell types, such as macrophages, are difficult to transduce (6). Furthermore, terminally differentiated cells often have a short turnover time and are replaced by precursor cells, causing rapid loss of transgene expres-

Conflict of interest: The authors have declared that no conflict of interest exists. Citation for this article: J. Clin. Invest. 120:379-389 (2010). doi:10.1172/JCI36666. sion. Identification of a long-lived cell type within a tissue compartment that can be efficiently and selectively transduced would provide a solution to several of the aforementioned problems.

Lung tissue is particularly accessible for gene transfer, since intraluminally instilled vectors readily gain access to the entire tracheobronchial tree. The 2 most common inherited lung diseases, cystic fibrosis and $\alpha 1$ antitrypsin deficiency-related emphysema, result from wellcharacterized single-gene defects that are theoretically correctable by durable transfer of genes (7-9). However, previous attempts to achieve robust and sustained gene expression in the respiratory tree have met with limited success $(1,3,10)$. Here we propose the alveolar macrophage (AM) as a localized target cell that can sustain expression of transferred genes in lung tissue (11). We present an intratracheally instilled lentiviral system able to selectively and efficiently deliver transgenes to murine AMs, which are resident in lung tissue at the time of transduction and continue to express transferred genes for at least 2 years. We apply what we believe is a novel approach to achieve sustained in vivo expression of normal human $\alpha 1$ antitrypsin (hAAT) protein at levels able to ameliorate emphysema in mice.

\section{Results}

Intratracheal instillation of lentiviral vectors results in durable intrathoracic gene expression. We previously reported that intratracheal instillation of lentiviral vectors resulted in intrathoracic reporter gene expression in live immunocompromised (nude; $\mathrm{Nu} / \mathrm{nu}$ ) 

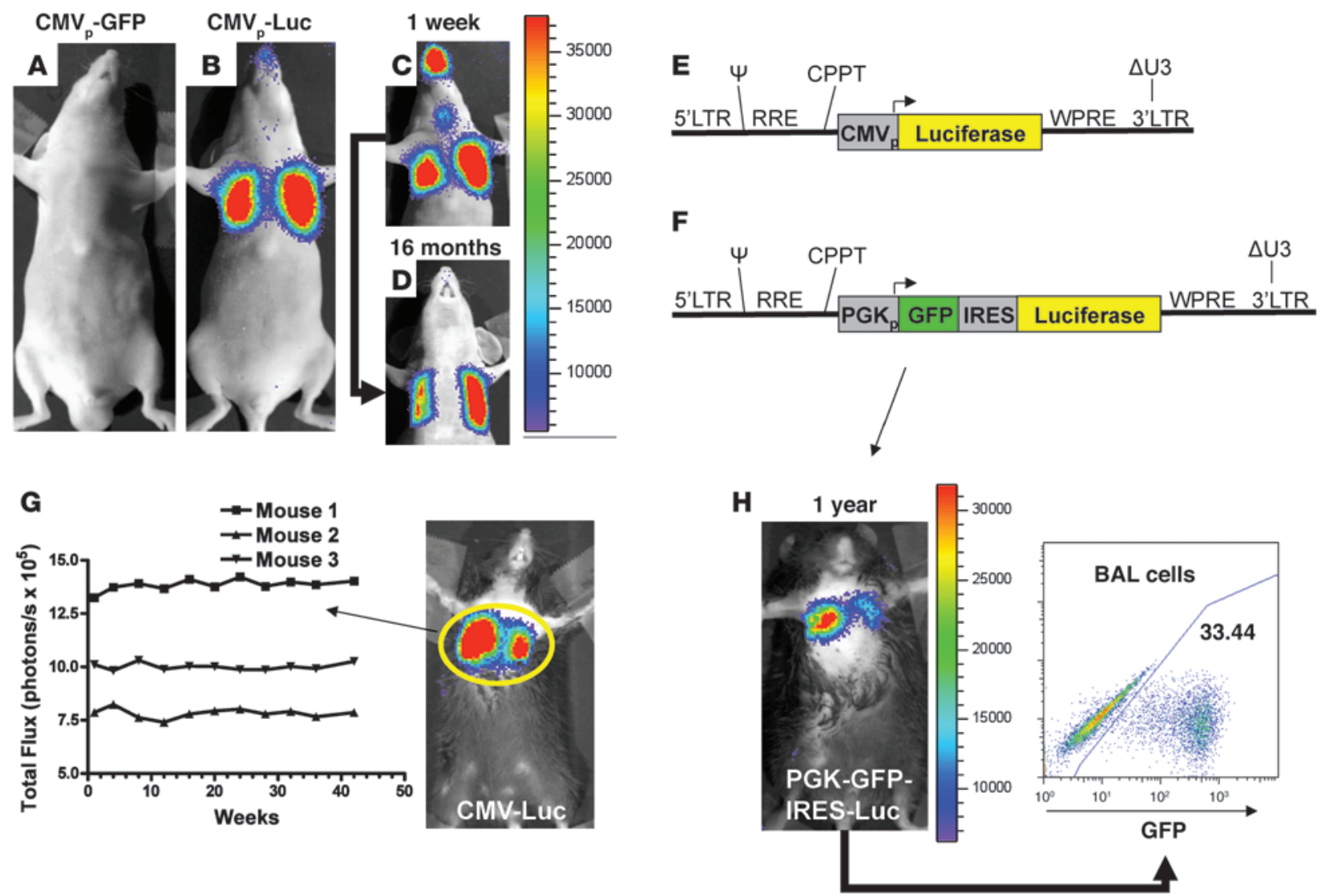

Figure 1

Kinetics of in vivo gene expression after lentiviral transduction. (A and B) Nude mice imaged by a CCD camera 5 days after intratracheal instillation of lentiviral vector carrying either a negative control (CMV-GFP) or luciferase reporter gene (CMV-luc). (C and D) Luciferase expression after a single lentiviral (CMV-luc) infection, imaged 1 week $(\mathbf{C})$ and 16 months (D) later in the same mouse. Note the persistent gene expression in the thorax. In contrast, gene expression observed in oropharyngeal and nasal regions in some recipients $(\mathbf{B}$ and $\mathbf{C})$ was transient. (E and $\mathbf{F})$ Schematic of lentiviral vectors CMV-luc (E) and PGK-GFP-IRES-Iuc (F). (G) Quantification of luciferase gene expression (total photon flux over the indicated thoracic region of interest) was followed for 42 weeks in three C57BL/6J mice after instillation of CMV-luc. Control mice injected with control vectors (CMV-GFP or CMV-lacZ) had no detectable photon flux above background. (H) Dual transgene expression 1 year after intratracheal instillation of a bicistronic lentiviral vector (PGK-GFP-IRES-luc). CCD camera imaging demonstrated in vivo expression of the luciferase reporter gene, while flow cytometry of cells obtained by BAL from this mouse demonstrated expression of GFP in $33 \%$ of cells. Pseudocolored heat maps indicate quantification of photons $/ \mathrm{s} / \mathrm{cm}^{2}$. LTR, lentiviral long terminal repeat; RRE, Rev responsive element; CPPT, central polypurine tract; WPRE, woodchuck hepatitis virus posttranscriptional regulatory element; $\Delta \mathrm{U} 3$, deleted U3 region for in vivo inactivation of the viral LTR promoter; IRES, internal ribosomal entry site.

mice imaged 5 days after infection (12). Recipients followed for the duration of their lives (16 months) unexpectedly revealed sustained reporter gene expression (Figure 1). Consequently, we sought to test the durability of gene expression in immunocompetent $(\mathrm{C} 57 \mathrm{BL} / 6 \mathrm{~J} ; n=3)$ mice. After a single intratracheal instillation of a lentiviral vector encoding a luciferase reporter transgene under control of CMV promoter regulatory elements (CMV-luc), we observed stable intrathoracic luciferase gene expression for a period of at least 42 weeks (Figure 1).

We employed histology and flow cytometry of lung tissue to determine the phenotype of transduced cells. Two months after infection with lentiviral vectors (CMV-GFP, $n=6$, or CMV-lacZ, $n=3$ ), frozen right lung sections revealed GFP or lacZ reporter gene expression exclusively in cells with the typical morphology and location of AMs resident in alveoli throughout the lung (Figure 2 and Supplemental Figure 1; supplemental material available online with this article; doi:10.1172/JCI36666DS1). These cells expressed the macrophage marker Mac-3 by immunostaining (Figure 2I). Transduction of airway or alveolar epithelium or endothelial cells was not detected, findings consistent with the known poor lung epithelial tropism in vivo of VSV-G pseudotyped lentiviruses (13-16).

Flow cytometry analysis of lung digests prepared from the contralateral lung of each CMV-GFP-treated mouse revealed that all $\mathrm{GFP}^{+}$cells expressed the hematopoietic marker CD45 (Figure $2 \mathrm{H}$ ). $\mathrm{GFP}^{+}$cells did not express the endothelial marker CD31 and did not express markers of differentiated lymphoid (B220, CD3) or myeloid (CD11b, GR-1) hematopoietic lineages (Figure $2 \mathrm{H}$ ). Because the known AM surface phenotype is $\mathrm{CD}^{-} 5^{+} \mathrm{GR} 1-\mathrm{B} 220^{-} \mathrm{CD} 3{ }^{-} \mathrm{CD} 31^{-} \mathrm{CD} 11 \mathrm{~b}^{-/ 10}(17,18)$, these results were consistent with our histologic observation that transduced cells were predominantly AMs. Lung epithelial, endothelial, fibroblast, and smooth muscle cells are known to be CD45(19-21), thus flow cytometry results confirmed that these cell types were not detectably transduced. 
A
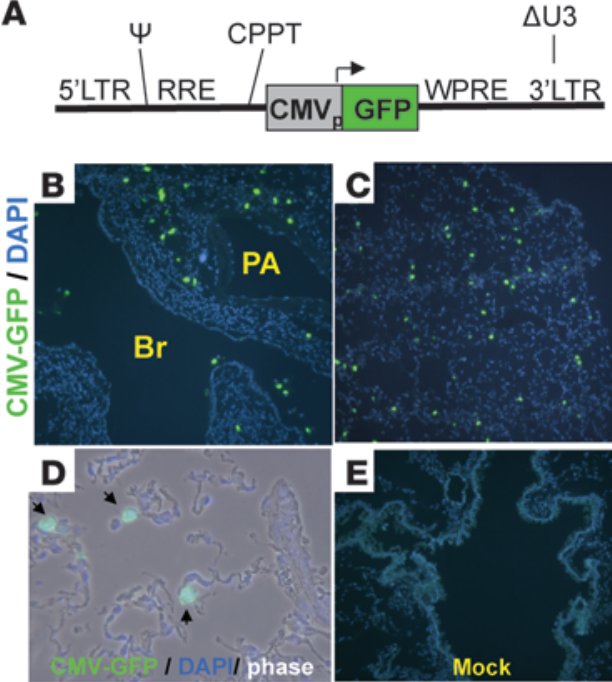

c

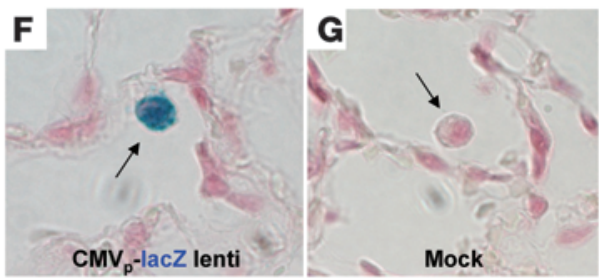

H
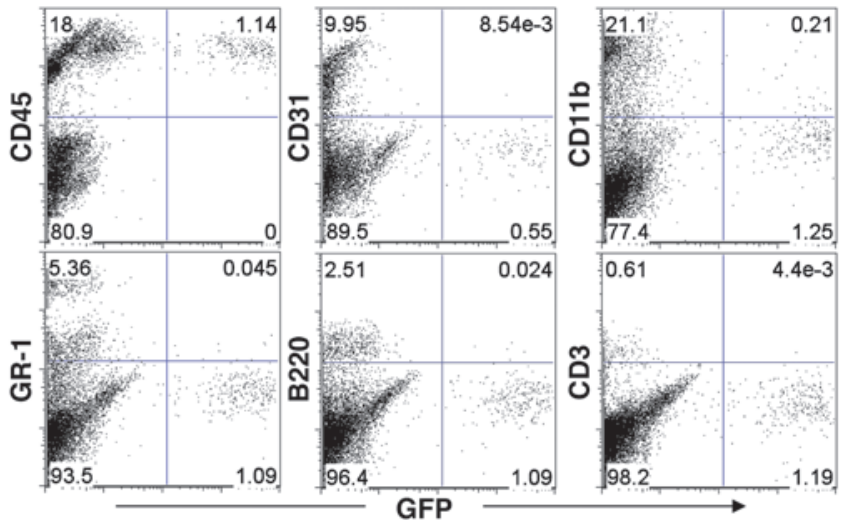

I
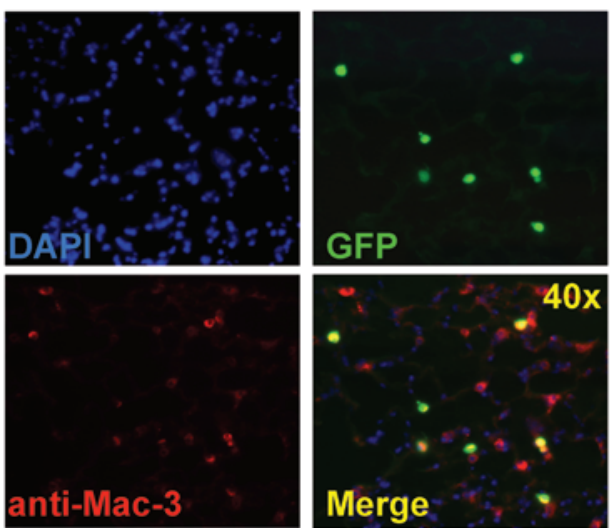

Figure 2

Phenotyping of transduced cells. Right lung (B-E) and left lung $(\mathbf{H})$ tissue from the same mouse assessed for GFP reporter gene expression 2 months after instillation of GFP-expressing lentiviral vector CMV-GFP (A). Fluorescence microscopy showed transduced (GFP+) cells in central/proximal (B) and distal (C) regions of the lung. Note that there was no transduction of airway epithelium or vascular endothelium (Br, bronchus; PA, pulmonary artery). (D) Overlay of GFP, DAPI, and phase-contrast images illustrated that GFP+ cells resided in alveolar air spaces and displayed the typical morphology of AMs. (E) Negative control lung after infection with mock lentivirus. (F and G) High-power view of X-galstained (blue) alveoli 2 months after transduction with CMV-lacZ or mock vectors. Arrows indicate AMs within the alveolar lumen of each mouse.

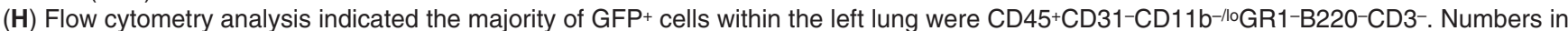
each quadrant indicate the percentage of cells in that quadrant. (I) Mac-3 (Cy3; red) immunostaining of lung after CMV-GFP transduction. GFP+ cells displayed positive anti-Mac-3 staining. Cell nuclei were counterstained with either DAPI (B-E and $\mathbf{I})$ or nuclear fast red (F and $\mathbf{G})$. Original magnification, $\times 10$ (B, C, and E), ×63 (D), ×100 (F and G). Additional images are available in Supplemental Figure 1.

Durable gene expression in vivo arises from long-lived quiescent $A M s$. In the steady state, AMs are the predominant hematopoietic cell type in lung alveoli and can be readily obtained from intact rodent or human lungs by the technique of bronchoalveolar lavage (BAL). Six weeks after infection with CMV-GFP lentivirus $(n=4$ per experiment, 3 repetitions; Figure 3 ), we observed GFP gene expression in approximately $40 \% \pm 15 \%$ (mean \pm SD) of live BAL cells. A gating algorithm (see Supplemental Figure 2) was used to analyze live AMs, which comprised more than $90 \%$ of all BAL cells. Optimal transduction efficiency of AMs occurred after delivery of $5 \times 10^{7}$ virions (Figure $3 \mathrm{I}$ ). As many as $70 \%$ of AMs were transduced at this dose. At higher virion doses, AM transduction efficiency did not increase, while the variance of transduction efficiency increased together with a 2-fold increase in MHC-II cell surface levels (Figure 3I and Supplemental Figure 3), suggesting that toxicity to AMs occurred when virion doses were administered beyond an optimal threshold. Cytospin analysis of only $\mathrm{GFP}^{+} \mathrm{BAL}$ cells purified by flow cytometry confirmed that all transduced cells (100 of 100) were AMs (Figure 3G). Furthermore,
$\mathrm{GFP}^{+} \mathrm{BAL}$ cells expressed the macrophage markers F4/80 (Figure $3 \mathrm{H}$ ) and $\mathrm{CD} 11 \mathrm{c}$ (data not shown), and more than $99 \%$ displayed AM forward/side scatter characteristics (Figure 3F). No GFP cells were found within gates comprising the $10 \%$ of non-macrophage BAL cell types (predominantly monocytes and lymphocytes). In addition, no $\mathrm{GFP}^{+}$cells were detected in the peripheral blood or bone marrow of any mouse analyzed 1-6 weeks after infection (Figure 4A).

We followed mice for up to 2 years after infection with either CMVGFP vector or bicistronic lentiviral vectors encoding both GFP and luciferase (Figures 1 and 3 ). In CMV-GFP-injected recipients $(n=4)$, $21.6 \% \pm 6 \%$ and $17.4 \% \pm 15.3 \%$ (mean \pm SD) of AMs obtained by BAL continued to express GFP after 1 and 2 years, respectively. All recipients treated with bicistronic vectors $(n=4)$ demonstrated sustained expression of both luciferase and GFP at 1 year (Figure 1).

Next, we considered the possibility that the prolonged presence of transduced AMs arose from the proliferation of either undifferentiated precursor cells or differentiated AMs. We used 2 weeks of continual in vivo BrdU exposure in order to label proliferating 

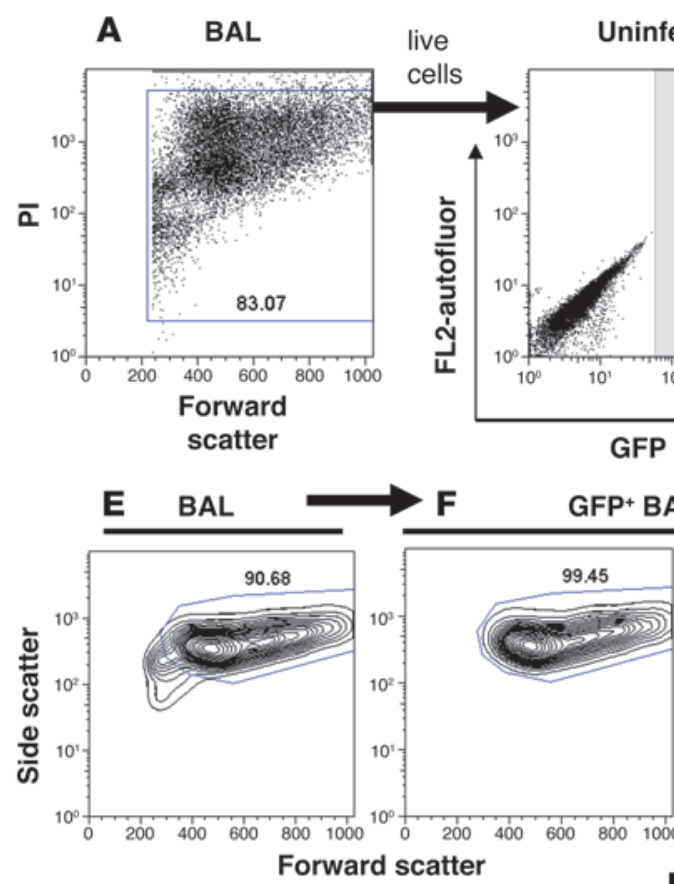

GFP

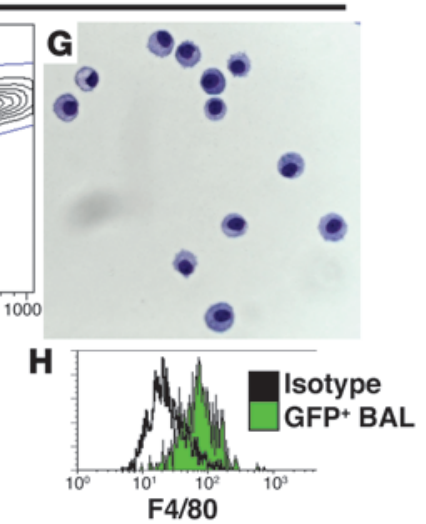

F $\quad$ GFP $^{+} \mathrm{BAL}$ cells

\begin{abstract}
B 6 weeks
\end{abstract}

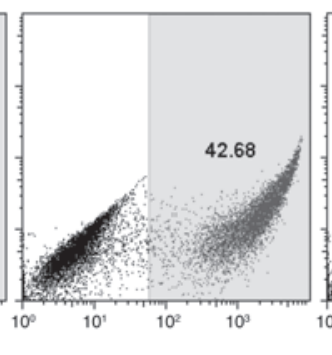

C

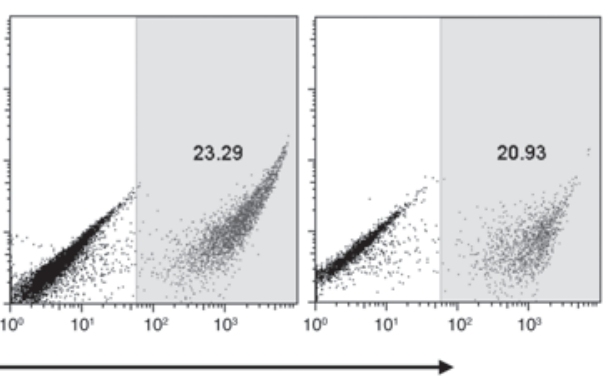

I

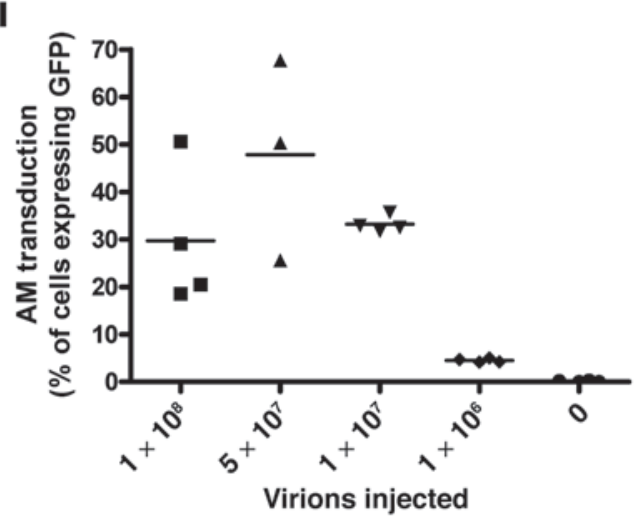

Figure 3

Analysis of AMs obtained by BAL after lentiviral transduction. (A) Flow cytometry of BAL cells obtained after transduction with CMV-GFP lentivirus showed that the majority of BAL cells were live (PI negative). (B-D) Representative dot plots of live (PI-negative) BAL cells showing the percentage of cells expressing the GFP reporter gene 6 weeks, 1 year, and 2 years after instillation of the CMV-GFP lentiviral vector. (E) Typical forward/side scatter flow cytometry profile of mouse BAL cells, with more than $90 \%$ of cells located within an AM gate. (F) Gating on only GFP+ BAL cells illustrating that more than $99 \%$ of GFP-expressing cells 6 weeks after transduction were located within the AM forward/side scatter gate. (G) Purification of GFP+ BAL cells by flow cytometry for cytospin analysis. GFP+ cells exhibited the characteristic Wright-Giemsa staining appearance of AMs. Original magnification, $\times 63$. (H) Flow cytometry histogram illustrating that GFP+ BAL cells expressed the macrophage marker F4/80 (black line overlay indicates isotype control staining). (I) Titering experiment illustrating transduction efficiency (percentage of GFP+ cells) of AMs obtained by BAL 6 weeks after instillation of the indicated quantity of CMV-GFP lentiviral particles. Each data point represents an individual mouse. Horizontal bars indicate the mean for each group. In $\mathbf{A}-\mathbf{F}$, the numbers within the plots denote the percentage of cells in the respective gated portion of the plot. A complete flow cytometry gating algorithm to identify live BAL AMs is provided in Supplemental Figure 2 .

cells in mice beginning 1 day after lentiviral CMV-GFP infection $(n=4$; Figure $4 \mathrm{~B})$. After 14 days, more than $70 \%$ of bone marrow cells in all mice were labeled with BrdU. In contrast, the 2-week labeling index of transduced $\mathrm{AMs}\left(\mathrm{GFP}^{+}\right)$, untransduced $\mathrm{AMs}$ $\left(\mathrm{GFP}^{-}\right)$, or unmanipulated AMs was $0.84 \% \pm 0.18 \%, 0.85 \% \pm 0.72 \%$, and $1.03 \% \pm 0.84 \%$, respectively (mean percentage of $\mathrm{BrdU}^{+}$cells $\pm \mathrm{SD}$ ). These results confirm the previously reported low proliferative index of steady-state AMs (22) and support our recently reported studies suggesting that the half-life of unperturbed AMs is essentially indistinguishable from the lifespan of mice (11). Taken together, the results demonstrate that proliferation of precursor cells is not the primary mechanism responsible for the prolonged presence of transduced AMs in our studies.

Inflammatory responses to lentiviral infection. Because intratracheal instillation of viral vectors can cause an inflammatory response, we performed BAL cell counts, cytospin differentials, and flow cytometry at $0,1,2$, and 6 weeks after lentiviral transduction ( $n=4$ per group; Figure $4, \mathrm{C}$ and D, and Supplemental Figure 4). At 1 week, we found evidence of mild pul- monary inflammation. BAL cell counts were elevated 2 -fold over baseline, and there were increased numbers of neutrophils $(10 \%-25 \%)$ and lymphocytes $(15 \%-25 \%)$ in BAL. This inflammatory response resolved significantly by 2 weeks after transduction; 6 weeks after transduction, BAL cell counts and differentials were indistinguishable from baseline levels.

To evaluate inflammatory activation of AMs, we assessed cell surface expression levels of $1 \mathrm{~A} / 1 \mathrm{E}$ MHC class II alloantigens by flow cytometry. Six weeks after infection with CMV-GFP lentivirus, we analyzed transduced $\left(\mathrm{GFP}^{+}\right)$and untransduced $\left(\mathrm{GFP}^{-}\right)$AMs from each mouse and compared expression levels of MHC-II with levels measured in AMs from unmanipulated control mice. MHC-II levels were identical to baseline when less than $10^{8}$ virions were initially delivered (Supplemental Figure 3). At higher viral doses, increased levels of MHC-II were present in all AMs (GFP ${ }^{+}$and $\left.\mathrm{GFP}^{-}\right)$ regardless of AM transduction status. For this reason, lentiviral doses less than $10^{8}$ were employed for all subsequent studies.

Lentiviral vectors transduce resident rather than recruited $A M s$. Based on the acute inflammatory response to lentiviral instillation, we 
BM: BrdU 2 weeks
BM: No BrdU

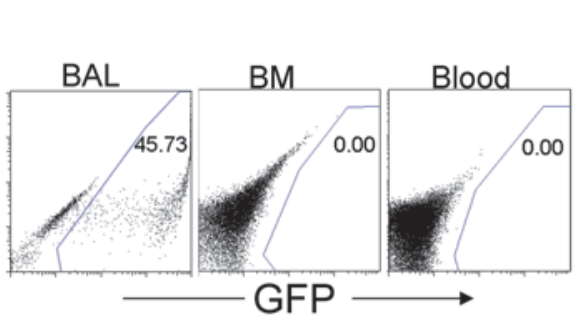

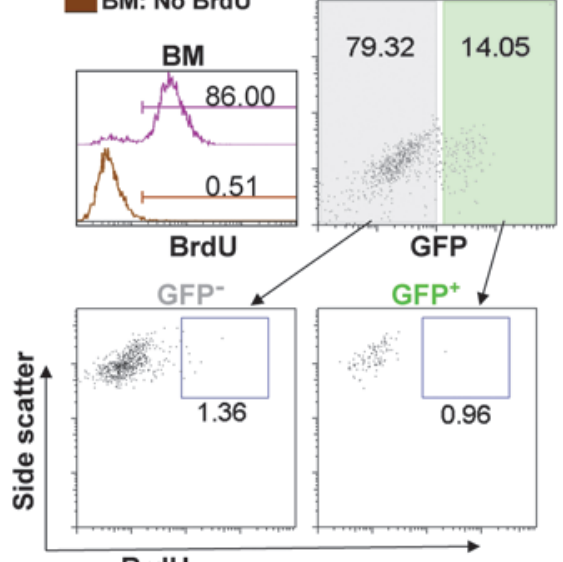

C

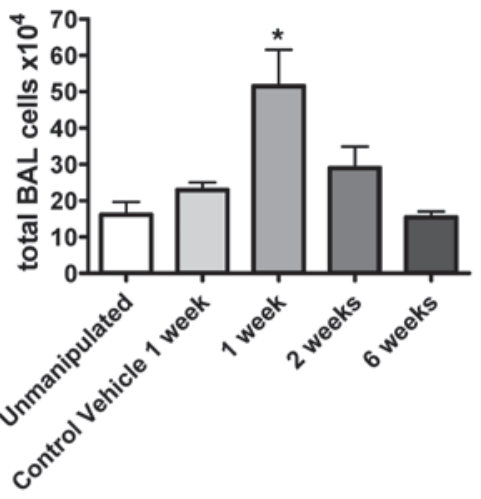

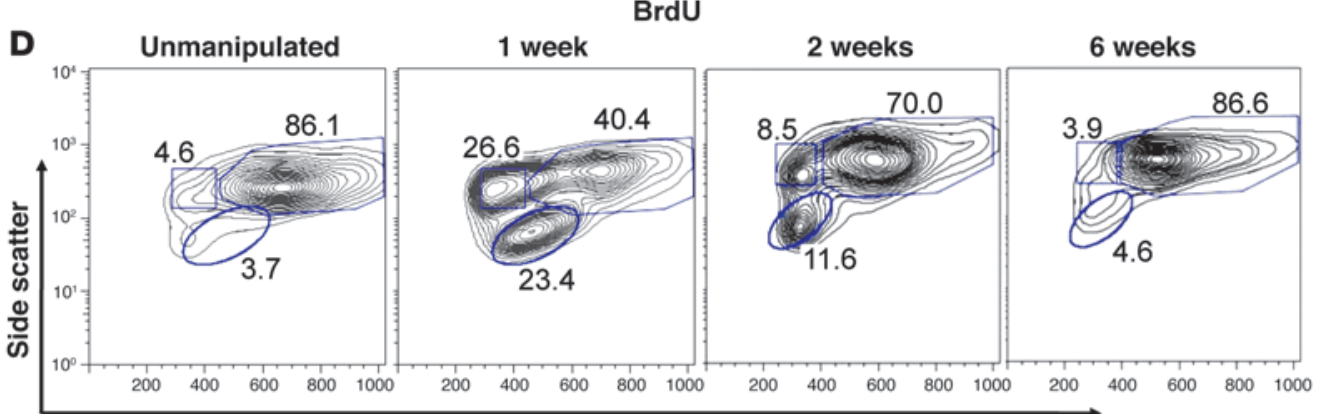

Forward scatter

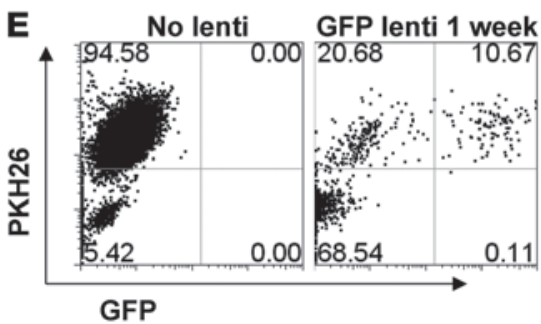

Figure 4

Quiescent transduced macrophages persist in lung tissue despite a transient acute pulmonary inflammatory response. (A) BAL, bone marrow, and blood cells from the same mouse analyzed for GFP expression 2 weeks after infection with CMV-GFP lentivirus. Note the transduction of BAL, but not bone marrow or circulating blood cells. (B) Bone marrow and AMs from mice infected with CMV-GFP and then exposed to BrdU for 2 weeks: $86 \%$ of marrow cells were BrdU labeled, compared with less than $2 \%$ of AMs, regardless of whether they were transduced (GFP+) or untransduced (GFP-). (C) BAL cell counts were increased above control 1 week after lentiviral infection, indicating a transient inflammatory response $\left(n=4 ;{ }^{*} P<0.05\right.$ by ANOVA). Data are presented as means \pm SEM. (D) Forward/side scatter analyses of BAL samples showed that more than $85 \%$ of BAL cells in normal mice were AMs. However, 1 week after intratracheal lentiviral instillation, there was an influx of neutrophils $(26 \%)$ as well as monocytes and lymphocytes (23\%; also quantified by cytospin differentials in Supplemental Figure 4). By week 6 , BAL forward/ side scatter profiles and cell counts were indistinguishable from baseline ( $n=4$ per time point). (E) A representative mouse treated with PKH26, infected the next day with CMV-GFP lentivirus, and analyzed 1 week later revealed that all transduced (GFP+) cells were resident in the lung at the time of infection (GFP+/PKH26+), whereas recruited inflammatory cells (68\% of BAL cells after infection) were PKH26- and GFP-. Numbers within the plots denote the percentage of cells in the gated portion or quadrant of the plot.

considered the possibility that recruited inflammatory cells, rather than resident AMs, might be transduced by lentiviruses. To test this, PKH26 fluorescent labeling of resident AMs was performed prior to lentiviral instillation. After a single PKH26 injection, this method allows tracking of resident AMs $\left(\mathrm{PKH} 26^{+}\right)$present in the lung at the time of labeling $(17,18,23,24)$. In contrast, peripheral blood cells and inflammatory cells recruited to the lungs after the time of labeling remain $\mathrm{PKH} 26^{-}$. Importantly, we found that all GFP-transduced BAL cells also were labeled with PKH26, indi- cating that only cells resident in the lung at the time of lentiviral instillation were transduced (Figure 4E).

Harnessing lentivirally transduced AMs for stable secretion of $h A A T$. The observation that resident AMs are long-lived cells able to achieve prolonged in vivo expression of transferred reporter transgenes suggests these cells as a novel vehicle for the durable expression of therapeutic transgenes. Hence, we adapted our lentiviral system to accomplish in vivo expression of hAAT selectively in AMs. We reasoned that this would result in secretion of hAAT directly into the 
A

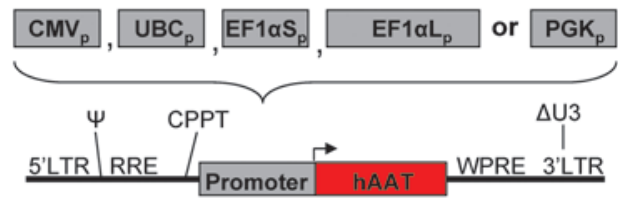

B

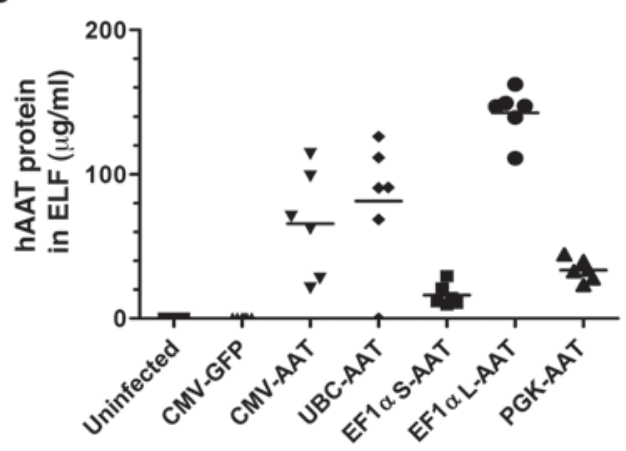

lung epithelial lining fluid (ELF), potentially providing protection against the development of emphysema. We utilized hAAT-expressing, VSV-G-pseudotyped lentiviral vectors that we had previously demonstrated as being able to (a) produce secreted hAAT protein of identical size to normal hAAT protein found in human serum and (b) secrete biologically active hAAT able to inactivate neutrophil elastase (25). Five different lentiviral constructs were tested in vivo in mice ( $n=6$ per group) in order to identify optimal promoter elements that can drive hAAT gene expression in vivo (Figure 5). Six weeks after intratracheal instillation of each vector, we observed that a 1.2-kb-long EF1 $\alpha$ promoter element (hereafter referred to as EF1 $\alpha$-hAAT) resulted in levels of hAAT in lung ELF above the protective threshold widely accepted in clinical trials as being potentially therapeutic in patients with hAAT deficiency (26). In these mice, hAAT protein secretion was highly localized to the lung, as evidenced by the presence of only trace circulating serum hAAT levels $(2.64 \pm 3.76 \mu \mathrm{g} / \mathrm{ml}$; detectable in only 3 of 30 mice).

To verify that no chronic inflammation resulted from administration of the EF1 $\alpha$-hAAT vector, we measured 19 cytokines in the BAL of each recipient by Bio-Plex cytokine array 6 weeks after infection with EF1 $\alpha$-hAAT vs. EF1 $\alpha$-GFP lentiviral vectors or control vehicle alone ( $n=6$ per group). In mice treated with either GFP- or hAATexpressing vectors, we observed all 19 cytokines to be at or below baseline levels compared with control vehicle-exposed mice (data not shown). Importantly, there was no evidence of either vector increasing levels of TNF- $\alpha$ or IL-6, key NF-KB-dependent cytokines believed to play a role in emphysema pathogenesis. Furthermore, there was no statistically significant difference between GFP- and hAAT-treated groups in 18 of 19 cytokines assayed (only MIP1a was suppressed in hAAT- compared with GFP-treated mice; $P<0.05$ ).

In vivo secretion of $h A A T$ does not activate humoral or cellular immune responses. We found expression of the hAAT transgene to be stable 12 and 24 weeks after intratracheal instillation of the EF1 $\alpha$-hAAT vector ( $n=4$ per group; data not shown). Because the prolonged expression of heterologous proteins can result in cellular and humoral immune responses, at 24 weeks we tested EF1 $\alpha$-hAATtreated mice for evidence of hAAT immunogenicity $(n=4$; Supplemental Figure 5). No circulating anti-hAAT IgG antibodies were detected in any recipient. Furthermore, after stimulation with hAAT protein, production of IL- 2 or IFN- $\gamma$ by $\mathrm{CD}^{+}$or $\mathrm{CD}^{+} \mathrm{T}$

\section{Figure 5}

In vivo lentiviral transfer of a hAAT gene. (A) Five different promoter fragments (CMV, UBC, 0.2-kb EF1 $\alpha$ [EF1 $\alpha \mathrm{S}], 1.2-\mathrm{kb}$ EF1 $\alpha$ [EF1 $\alpha \mathrm{L}]$, and $P G K)$ were ligated into the illustrated lentiviral vector and administered to mice 6 weeks prior to ELISA-based assessment of hAAT protein levels in lung ELF. (B) The 1.2-kb EF1 $\alpha$ promoter fragment resulted in the highest levels of secretion of hAAT protein. Each data point represents an individual mouse. Horizontal bars indicate the mean for each group; $n=6$ per group.

cells isolated from the spleens of treated animals was no different than that observed in naive or unstimulated control cells (Supplemental Figure 5). Similarly, there was no detectable $\mathrm{T}$ cell proliferation by CFSE assay (data not shown).

Lentiviral-mediated expression of hAAT ameliorates elastase-induced emphysema. Next, we tested the ability of intratracheally delivered lentiviral vectors to ameliorate emphysema. Intratracheal instillation of porcine pancreatic elastase (PPE) into rodents is an established model of emphysema, resulting within 21 days in sustained airspace enlargement, increased heterogeneity of alveolar size, and increased lung compliance reminiscent of human pulmonary emphysema (27-29). In order to quantify the severity of elastaseinduced emphysema in mice, we performed physiologic assessments of lung function on anesthetized mice as well as histological analyses of air space structure.

Mice treated with intratracheally instilled EF1 $\alpha$-hAAT lentivirus (vs. EF1 $\alpha$-GFP control; $n=15$ per group) were maintained for 8 weeks to allow stable gene expression and resolution of acute inflammation. Emphysema was then induced by intratracheal instillation of PPE vs control vehicle (PBS), and all animals were analyzed 21 days later (Figure 6). In mice exposed to control vehicle, both lung physiology and histology were normal with no detectable difference between EF1 $\alpha$-GFP- and EF1 $\alpha$-hAAT-treated mice (Figure 6, E and J). Hence, these data were combined (hereafter referred to as PBS-control) for further analysis. In EF1 $\alpha$-GFP-treated mice, PPE exposure resulted in emphysema as detected by all measured physiologic and histologic parameters, including significantly increased lung compliance (Figure 6F), equivalent alveolar diameter (Figure 6G), SD of alveolar diameter, an indicator of heterogeneity (Figure 6H), and the area-weighted mean alveolar diameter (an index sensitive to changes in both airspace enlargement and its heterogeneity) (Figure 6I) (28). However, as summarized in Figure 6, EF1 $\alpha$-hAAT-expressing mice showed statistically significant amelioration of elastase-induced emphysema compared with mice treated with EF1 $\alpha$-GFP. hAAT gene transfer decreased equivalent alveolar diameter to normal levels while significantly ameliorating the effects of elastase on lung compliance and the area-weighted mean alveolar diameter. Notably, changes in alveolar structure characterized by the area-weighted mean alveolar diameter correlated well with changes in compliance $(P<0.001$; Figure $6 \mathrm{~J})$.

The majority of elastase-induced emphysema develops between 5 and 21 days after PPE exposure and depends, in part, on the presence of pulmonary inflammation, including increased numbers of recruited macrophages $(29,30)$. To assess whether hAAT overexpression affects the kinetics of the inflammatory response to PPE, we analyzed mouse BALs 3, 7, and 21 days after PPE instillation (Figure 6). We found that overexpression of hAAT did not simply act by abrogating any inflammatory effect of the instilled elastase, since both EF1 $\alpha$-hAAT- and EF1 $\alpha$-GFP-treated groups exhibited a brisk acute inflammatory response within 3 days, as evidenced 

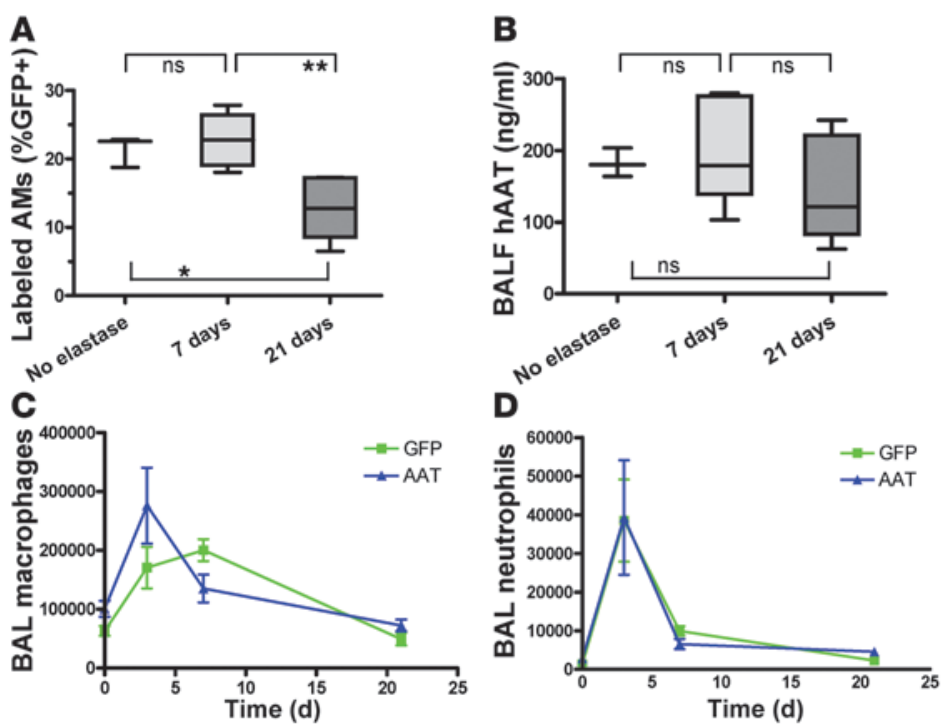

\section{$\mathbf{E}$}
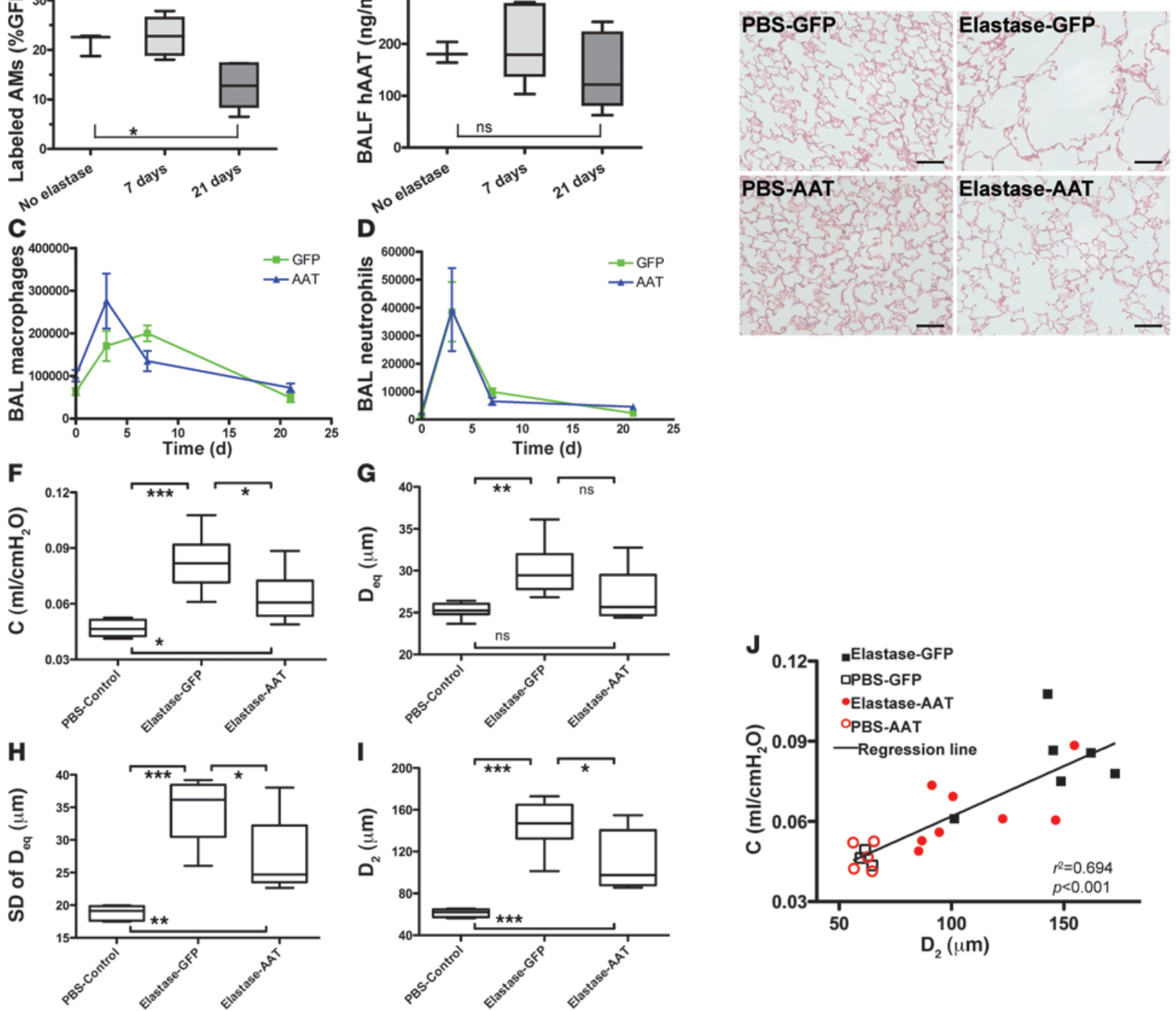

Figure 6

Amelioration of elastase-induced emphysema in mice treated with EF1 $\alpha$-hAAT lentivirus. (A-D) Analysis of BAL cells and BAL fluid (BALF) from mice treated with EF1 $\alpha$-GFP versus EF1 $\alpha$-hAAT lentiviral vectors followed by intratracheal elastase. (A) In mice treated with EF1 $\alpha-G F P$ lentiviral vector, the percentage of AMs in the BAL that expressed GFP is shown for each time point after elastase exposure. (B) hAAT protein levels in the BAL fluid of EF1 $\alpha$-hAAT-treated mice following elastase exposure. (C and D) Kinetics of macrophage recruitment (C) and neutrophil recruitment (D) following elastase exposure. Data are represented as means \pm SEM. (E) Representative hematoxylin and eosin-stained lung sections from the indicated groups 21 days after elastase exposure. Scale bars: $100 \mu \mathrm{m}$. (F-I) Comparison of lung compliance (C), equivalent alveolar diameter $\left(D_{\text {eq }}\right)$, heterogeneity $\left(S D\right.$ of $\left.D_{\text {eq }}\right)$, and area-weighted mean alveolar diameter $\left(D_{2}\right)$, a parameter sensitive to changes in both airspace enlargement and its heterogeneity (28). (J) Structure-function relationship demonstrating that increases in $D_{2}$ correlated with increases in $\mathrm{C}\left(r^{2}=0.694\right)$. Note that there was no difference between the control groups of PBS-exposed mice treated with either GFP- or AAT-expressing vectors. The graph in $\mathbf{J}$ combines the data in $\mathbf{F}$ and $\mathbf{I}$. Box plots in $\mathbf{A}, \mathbf{B}$, and $\mathbf{F}-\mathbf{I}$ illustrate median (horizontal line), 25th and 75 th percentiles (upper and lower box boundaries), and highest and lowest observed values (whiskers). ${ }^{*} P<0.05 ;{ }^{* *} P<0.01$; ${ }^{* * *} P<0.001$ (ANOVA with post-hoc Tukey's multiple comparisons test).

by rapid recruitment of neutrophils into lung airspaces. Rather, the EF1 $\alpha$-hAAT-treated group exhibited an altered macrophage inflammatory kinetic response to elastase over time. The elevated number of lavagable macrophages in the lung appeared to peak earlier and resolve faster in the EF1 $\alpha$-hAAT-treated group. In contrast, there was no difference in the kinetics of the neutrophilic inflammatory response to elastase in either group.
Because recruitment of macrophages into lung airspaces following PPE instillation resulted in a rapid increase in lavagable cell numbers, followed by gradual resolution back to baseline over 21 days (Figure 6), we considered the possibility that PPE had caused the replacement of lentivirally transduced AMs in lung airspaces. In EF1 $\alpha$-GFP-treated mice subsequently exposed to PBS compared with elastase, GFP expression remained in $21 \% \pm 2 \%$ vs. $13 \% \pm 5 \%$ of 
BAL AMs, respectively, at 21 days (corresponding to an average of $13,437 \pm 3,075$ vs. $5,932 \pm 2,990 \mathrm{GFP}^{+}$lavaged AMs, respectively). Similarly, in EF1 $\alpha$-hAAT-treated mice, secreted hAAT protein persisted in the BAL fluid at all time points, regardless of exposure to elastase (Figure 6). Thus, lentivirally transduced AMs persisted in lung tissue 21 days after elastase exposure despite the induction of emphysema, although the percentage and number of all AMs expressing GFP was lower than in the PBS-exposed group.

Next, we sought to examine the effects of EF1 $\alpha$-hAAT treatment in an additional model of pulmonary inflammation that does not depend on elastase instillation. We employed an established model of pulmonary inflammation induced by acute tobacco smoke exposure (31) in groups of mice pretreated with either EF1 $\alpha$ hAAT or control EF $1 \alpha$-GFP lentiviral vectors $(n=10$ animals per group). Again, we noted the surprising persistence of lentivirally transduced AMs in lung lavage fluid, even after the induction of acute smoke-induced pulmonary inflammation (Supplemental Figure 6). Similar to our findings in the PPE model, in 2 repeated experiments we found that hAAT overexpression did not significantly dampen the recruitment of inflammatory cells into the lung lavage fluid, arguing against a broad primary anti-inflammatory effect resulting from hAAT overexpression. Furthermore, a statistically significant amelioration of smoke-induced cytokine induction resulting from hAAT overexpression was only observed on 1 of 5 measured BAL fluid cytokines (KC; $P=0.047$ ), with no effect observed on TNF- $\alpha$, IL- 6 , or IL- $1 \mathrm{~b}(P=0.46,0.17$, and 0.13 , respectively) and a nonsignificant, marginal effect on MCP-1 $(P=0.07)$. Taken together, our findings indicate that localized overexpression of hAAT in lung tissue ameliorates elastase-induced emphysema but does not broadly abrogate pulmonary inflammatory responses to instilled elastase or to acute tobacco smoke inhalation.

\section{Discussion}

In this study we establish a method for efficiently and selectively delivering genes to resident AMs in vivo, and we apply this method for gene therapy of emphysema. Although macrophages have been considered difficult to efficiently transfect either in vitro or in vivo $(6,32)$, we demonstrate that VSV-G-pseudotyped lentiviral vectors efficiently transduced AMs, which persisted for at least 2 years in the airspaces of mouse lung.

We have adapted our method to durably overexpress hAAT in AMs - a key cell type known to be involved in emphysema pathogenesis (33). Considerable effort has been invested in attempts to deliver a normal hAAT gene in vivo $(7,9,34,35)$. Many of these approaches have been limited by either short-lived gene expression or inability to achieve the very high levels of circulating hAAT $(1 \mathrm{mg} / \mathrm{ml})$ presumably required to prevent the progression of emphysema in hAAT-deficient patients $(7,26,36,37)$. An important concept in hAAT gene therapy is the likelihood that localized secretion of hAAT by cells within the lung may provide more effective protection against emphysema at hAAT levels far lower than those required in the circulating blood $(7,37)$. Our findings support this concept, since intratracheal delivery of lentivirus provided therapeutic localized secretion of hAAT into the lung ELF in the absence of high circulating concentrations of hAAT protein. Our method achieves an average lung ELF hAAT protein concentration of $142 \mu \mathrm{g} / \mathrm{ml}$, equivalent to a $42 \%$ increase in total AAT over estimated endogenous mouse ELF AAT protein levels (refs. 38-40 and Supplemental Methods). The vectors presented in our mouse studies should be readily translatable to human cells, as we have observed efficient transduction and gene expression in primary human AMs after in vitro lentiviral infection (46\% GFP transduction efficiency; Supplemental Figure 7).

The current standard of care for patients with severe emphysema and hAAT deficiency consists of life-long weekly injections of hAAT protein purified from pooled human plasma (26). Whether this approach is clinically effective or results in detectable inhibition of lung matrix breakdown in patients remains controversial $(41,42)$. In mouse models of emphysema, injected hAAT protein has been shown to ameliorate tobacco smoke-induced emphysema via an anti-inflammatory mechanism related to TNF- $\alpha$ suppression $(43,44)$. In addition, in mice exposed to VEGF receptor blockade, hAAT protein has been shown to attenuate airspace enlargement through inhibition of apoptosis and modulation of lung oxidant stress (45). Although our results did demonstrate altered macrophage recruitment kinetics after elastase injury, further investigations will be required to determine whether this altered inflammatory kinetic or the emphysema amelioration in our studies resulted predominantly from the well-known antiprotease effects or other aforementioned properties of hAAT.

While our protocol offers the prospect of sustained hAAT reconstitution using a single lentiviral-based treatment, our experiments tested augmentation of AAT in mice that already express normal murine AAT. Because complete absence of AAT in mice results in embryonic lethality (46), a murine model of severe AAT deficiency that resembles the human disease is not currently available. Future tests of the efficacy of our protocol in inducible AAT-knockout mice should help to further define the therapeutic potential of our approach. In addition, before clinical use can be considered, more detailed screens for adverse consequences, including insertional mutagenesis of transduced cells, will be required.

Our findings challenge the widely accepted view of AMs as being short-lived cells which require continual replenishment by circulating bone marrow-derived monocyte precursors $(22,47)$. The low BrdU labeling index of AMs in the steady-state lung, the persistence of lentivirally tagged cells on kinetic imaging studies, and the absence of appreciable numbers of transduced circulating cells in our experiments all support the conclusion that a prolonged AM lifespan, rather than proliferation or recruitment of precursor cells, explains the unexpectedly long duration of these cells in uninjured lung tissue. Our findings differ from most prior studies of AM lifespan because the majority of prior reports have relied on myeloablative regimens to examine the kinetics of AM reconstitution with transplanted circulating or bone marrow-derived cells $(22,47,48)$. Those kinetics likely reflect the toxic effects of myeloablative conditioning regimens rather than the true lifespan of AMs. Indeed, we recently reported that when AMs are shielded from radiation exposure in recipient mice being prepared for bone marrow transplantation, there is minimal AM turnover even 8 months after reconstitution of the bone marrow and blood (11). Because the durable gene expression achieved in our current study depends on the prolonged lifespan of unperturbed murine AMs, it will be important for future studies to determine whether similar longevity applies to AMs in the lungs of humans with or without lung disease. Our results demonstrating durable gene expression in transduced AMs even following PPE- or acute tobacco smoke-induced injuries suggests that resident AMs similarly persist in diseased lungs, although whether AM proliferation in inflamed lungs contributes to this persistence was not assessed in our studies. 
The preferential tropism of VSV-G-pseudotyped lentiviruses for AMs in our studies likely results from the comparatively poor tropism of this pseudotype for the other major luminal constituent of the lung, epithelial cells. Receptors for the VSV-G envelope are thought to be sequestered on the basolateral surface of adult lung epithelia, preventing their in vivo transduction in the absence of epithelial injury in most prior reports (13-16). However, VSV-Gpseudotyped lentiviral vectors do appear to possess some capacity to transduce lung epithelial cells in vitro $(49,50)$, when administered in vivo at early fetal developmental stages $(51,52)$, or when delivered together with epithelial mitogens such as KGF (53). Alternatively, lentiviral vectors pseudotyped with other viral envelope glycoproteins such as Ebola do appear to possess adult lung epithelial tropism in vivo $(13,15,52,54)$. We have found that Ebola-pseudotyped (NTDL6; ref. 54) lentiviruses also efficiently transduce AMs in vivo but lack the AM specificity of the VSV-G pseudotype (our unpublished observations).

It cannot be concluded from our studies that lentiviral transduction was entirely specific to AMs. For example, some animals showed persistent gene expression at the tip of the nose (Figure 1). In addition, transient reporter gene expression was occasionally observed over the oropharyngeal region, implying that extrathoracic cell types such as pharyngeal lymphoid tissue may have expressed transferred genes. Indeed, the acute intra-alveolar inflammatory response observed 1 week after lentiviral instillation indicates significant inflammatory signaling and activation of a variety of inflammatory cell types, some of which are likely to transiently traffic through the lymph or blood to sites outside the lung. That pulmonary dendritic cells in particular may have been transduced in our studies cannot be excluded because these cells would be expected to migrate to local draining lymph nodes within hours of infection. It is also important to note that we did not find evidence of preferential lentiviral transduction of any particular AM subset (as defined by degree of quiescence, or expression levels of cell surface MHC-II, CD11b, CD11c, or F4/80). However, our results do not entirely exclude the possibility that a particular, as yet unrecognized, subset of lung macrophages may have been preferentially transduced by our method.

In summary, our results suggest the feasibility of selectively targeting differentiated cells resident in a tissue to accomplish prolonged and therapeutic in vivo gene expression. The method presented here should be of considerable interest to those wishing to modulate gene expression in AMs for the study of pulmonary innate immunity or the pathogenesis of a variety of inflammatory lung diseases, including asthma and emphysema.

\section{Methods}

Animal studies and luciferase imaging. C57BL/6J or $\mathrm{Nu} / \mathrm{J}$ (nude; nu/nu) 8- to 12-week-old mice (The Jackson Laboratory) were maintained in a pathogen-free facility. For in vivo BrdU labeling studies, selected C57BL/6J mice were given BrdU (BD Biosciences) in their drinking water $(0.8 \mathrm{mg} / \mathrm{ml})$ daily for 14 days, beginning the day after lentiviral infection. All animal studies were approved by the Institutional Animal Care and Use Committee of Boston University School of Medicine.

Vectors and viral production. Replication incompetent lentiviruses used in the gene transfer experiments were created using a 5-plasmid transfection procedure $(12,25)$. Briefly, 293 T cells were transfected with the pHAGE backbone lentiviral vector (12) together with 4 expression vectors encoding the packaging proteins Gag-Pol, Rev, Tat, and the G protein of the vesicular stomatitis virus (VSV-G). The pHAGE lentiviral backbone used in all experiments was an optimized self-inactivating lentiviral long-terminal repeat, nonreplicative vector derived from the original pHR'CMVlacZ vector previously described by Naldini et al. (55). The backbone contains the woodchuck hepatitis virus posttranscriptional regulatory element, which enhances levels of transcription and gene expression (56). Cloning procedures used to generate pHAGE lentiviral backbone plasmids containing each indicated transgene (luciferase, enhanced GFP, lacZ, or hAAT; ref. 25) or each promoter element (CMV, PGK, UBC, or $\mathrm{EF} 1 \alpha)$ are available in the Supplemental Methods. All viral supernatants were concentrated approximately 100 -fold by ultracentrifugation. Titering of all vectors was performed by infection of FG293 cells as previously published (25), and viral titers of about $5 \times 10^{8}$ to $5 \times 10^{9} / \mathrm{ml}$ were typically achieved with this protocol.

In vivo transduction. Mice were anesthetized with isoflurane. Concentrated lentiviral supernatant $(100 \mu \mathrm{l})$ was delivered via a blunt-ended, 18-gauge needle into the posterior oropharynx just above the tracheal entrance. Aspiration of this fluid bolus upon the subsequent inhalation was confirmed in each recipient by investigator visualization. Thirty minutes prior to injection, all viral supernatants were mixed with Lipofectamine 2000 (5\% final vol/vol; Invitrogen) in order to achieve a 2 -fold increase in transduction efficiency.

Noninvasive bioluminescence imaging. Prior to imaging, mice were anesthetized and injected with $150 \mu \mathrm{l}$ luciferin substrate $(30 \mathrm{mg} / \mathrm{ml}$; Xenogen). A series of bioluminescent images were then taken for up to 30 minutes using a Xenogen IVIS 100 imager. Photon output was quantified at the plateau of the time course using Living Image software (Xenogen).

Fluorescence microscopy. For analysis of GFP expression in lung tissue, the right lungs of mice were inflation fixed overnight with $4 \%$ paraformaldehyde and embedded in OCT. Frozen sections $5 \mu \mathrm{m}$ thick were covered with DAPI-containing mounting media (Vector labs) and analyzed by fluorescence microscopy to examine green (GFP) and blue (DAPI) fluorescence. Phase contrast microscopy of each field was used to assess the morphology and location of $\mathrm{GFP}^{+}$cells. Mac- 3 immunostaining was performed with biotinylated monoclonal anti-mouse Mac-3 antibody (BioLegend clone M3/84; diluted 1:100) or biotinylated isotype control, followed by second step staining with streptavidin-conjugated Cy3.

$B A L$. After euthanasia, BAL was performed by cannulation of the trachea and instillation and aspiration of consecutive aliquots of PBS. Depending on the experiment, two or three 500- $\mu$ l (for 1-lung lavage) or 1,000- $\mu$ l (for 2-lung lavage) aliquots were instilled. BAL cells and fluid were separated by centrifugation. BAL fluid was stored at $-80^{\circ} \mathrm{C}$ for analysis of hAAT protein, cytokines, and urea content.

Flow cytometry. Single-cell suspensions of lung tissue were prepared by enzyme digestion with collagenase A and dispase II (Roche), as previously described (19). Single-cell suspensions or BAL cell pellets were stained with fluorescence-conjugated anti-mouse monoclonal antibodies against CD45 (clone 6B2), CD31 (MEC13.3), CD11b (M1/70), GR-1 (RB6-8C5), B220 (RA36B2), CD3 (145-2C11), MHC-II 1A/1E (M5/114.15.2; all from BD Biosciences), or F4/80 (clone BM8; eBioscience). Gating thresholds to identify positively stained cells were determined by staining parallel cell aliquots with monoclonal antibodies of identical isotype. Propidium iodide (PI; $2 \mu \mathrm{g} / \mathrm{ml}$; Molecular Probes) was added to all non-fixed samples to exclude dead cells from analysis. A gating algorithm (forward/side scatter high, autofluorescence high, and PI negative; illustrated in Supplemental Figure 4) was used to identify live AMs within BAL cell populations. For BrdU staining, paraformaldehyde-fixed BAL or bone marrow cells were stained with APC-conjugated anti-BrdU antibody (BrdU Flow Kit; BD Biosciences). In vivo staining of macrophages with PKH26 (Red Fluorescent Phagocytic Cell Linker Kit; Sigma-Aldrich) was performed by intravenous injection of $100 \mu \mathrm{l}$ of PKH26 at a 1:5 dilution. Blood or BAL cells from PKH26-treated mice were harvested 1 week later for analy- 
sis. All flow cytometry sorting or analyses were performed on MoFlo (DakoCytomation), BD FACScan, or BD LSR II (BD Biosciences) instruments.

hAATELISA. hAAT protein concentrations in blood and BAL fluid specimens were determined by ELISA (25). hAAT was specifically captured with rabbit anti-human AAT antibody (Sigma-Aldrich) and detected with horseradish peroxidase-conjugated goat anti-human AAT antibody (EY Laboratories; protocol provided by Roberto Calcedo and Joanita Figueredo, University of Pennsylvania, Philadelphia, Pennsylvania, USA). The lower limit of detection for hAAT was $0.39 \mathrm{ng} / \mathrm{ml}$. After quantitation of hAAT concentrations in BAL fluid, concentrations of hAAT in lung ELF were calculated using the urea dilution method developed by Rennard et al. (57) (Quantichrom Urea Assay; Bioassay Systems). For more information, see Supplemental Methods.

Cytokine profile analysis. BAL fluid aliquots from mice exposed to lentiviruses versus the control vehicle were analyzed for levels of 19 cytokines by Bio-Plex cytokine array (Bio-Plex 23 cytokine reagent kit with Bio-Plex mouse cytokine panel in the Bio-Plex 200 system; Bio-Rad) as previously described (58). The estimated level of each cytokine in lung ELF was then calculated using the urea dilution method $(25,57)$. The cytokines assayed were TNF- $\alpha$, IFN- $\gamma$, IL-1b, IL-2, IL-4, IL-5, IL-6, IL-9, IL-10, IL-12(p70), IL-12(p40), IL-13, IL-17, G-CSF, KC, MCP-1, MIP1a, MIP1b, and Rantes. Additional BAL fluid samples from lentivirus-treated mice exposed to cigarette smoke were analyzed by 5-plex multiplex array (Milliplex MAP mouse cytokine kit; Millipore). Cytokines measured in these experiments (TNF- $\alpha$, IL-1b, IL-6, KC, and MCP-1) were not corrected for dilution.

Immune response. Serum and splenocytes from mice were collected 24 weeks after treatment with intratracheal lentivirus. Sera were assessed for circulating anti-hAAT-specific IgGs by ELISA as previously published (25). T cells from splenocyte samples were stimulated by incubation with anti-CD28 $(2 \mu \mathrm{g} / \mathrm{ml})$, anti-CD 49d $(2 \mu \mathrm{g} / \mathrm{ml})$, and hAAT whole protein $(4 \mu \mathrm{g} / \mathrm{ml})$. Unstimulated cells were incubated in the above reagents minus the protein, while positive controls were incubated with PMA (SigmaAldrich; $2 \mu \mathrm{g} / \mathrm{ml})$ and ionomycin $(10 \mu \mathrm{g} / \mathrm{ml})$. Following stimulation, intracellular cytokine staining of splenocytes was performed as published using fluorescence-conjugated monoclonal antibodies specific to CD4, $\mathrm{CD} 8$, IFN- $\gamma$, and IL-2 (59). The percentage of $\mathrm{CD} 4^{+}$and $\mathrm{CD} 8^{+}$lymphocytes producing IFN- $\gamma$ and IL- 2 in response to hAAT stimulation was quantified by flow cytometry. For more information, see Supplemental Methods.

Emphysema model. C57BL/6J mice (The Jackson Laboratory) were first treated with hAAT- or GFP-expressing lentiviral vectors. Eight weeks after treatment, animals received intratracheally instilled PPE $(0.28 \mathrm{U}=30 \mu \mathrm{g}$; Sigma-Aldrich) dissolved in $100 \mu \mathrm{l}$ of saline or $100 \mu \mathrm{l}$ of saline alone (control vehicle). At 3, 7, and 21 days after elastase, BALs were performed and analyzed by cell count, cytospin, FACS (for GFP expression), hAAT ELISA, and multiplex cytokine array. Lung physiological and histological measurements were performed at the 21-day time point.

Measurements of lung physiology. Mice were anesthetized with pentobarbital and tracheostomized, and the tracheal cannula was connected to a computer-controlled ventilator (Flexivent; SCIREQ). Respiratory mechanics were measured using the forced oscillation technique (60). Respiratory impedance spectra obtained using Fourier analysis were fitted to a model that included airway resistance, tissue damping, and tissue elastance $(\mathrm{H})$. We report here the inverse of $\mathrm{H}$, which is respiratory compliance (C). Since the chest of the mouse is very compliant (60), C is essentially lung compliance. For more information, see Supplemental Methods.

Quantification of histology. The lungs were inflation-fixed overnight with $4 \%$ paraformaldehyde at an airway pressure of $25 \mathrm{cmH}_{2} \mathrm{O}$. Fixed lungs were embedded in paraffin by standard methods, sectioned at $5 \mu \mathrm{m}$, and stained with hematoxylin and eosin. The equivalent diameters of alveolar airspaces were obtained from digital images (28), and the area-weighted mean alveolar diameter index was computed. This index has been shown to be sensitive to changes in both airspace enlargement and its heterogeneity (28). For more information, see Supplemental Methods.

In vivo cigarette smoke exposure. Female C57BL/6J mice ( $n=10$ per group) were pretreated with hAAT- or GFP-expressing lentiviral vectors 8 weeks prior to tobacco smoke exposure. Smoke exposure was performed 4 times a day, 5 days per week for 2 weeks in a nose-only exposure chamber (61). Each exposure employed whole smoke from 1 University of Kentucky 3R4F cigarette with the filter removed. Negative control mice treated with each vector ( $n=6$ per group) were exposed to room air.

Statistics. One-way ANOVA with post-hoc Tukey's multiple comparison testing was used to compare histologic, physiologic, and cytokine measurements between the indicated groups. Where indicated for tobacco smoke exposure studies, the 1-tailed Student's $t$ test was used to compare specified groups. In all studies, differences between groups were considered statistically significant at $P<0.05$.

\section{Acknowledgments}

This study was supported by the following grants: 5R21HL086414 (to D.N. Kotton), Alpha-1 Foundation/American Thoracic Society A-05-005 (to D.N. Kotton), HL-59215 (B. Suki), and Flight Attendant Medical Research Institute YCSA\#062572 (to A.A. Wilson). The authors wish to thank Mary C. Williams, Jerome S. Brody, and Gustavo Mostoslavsky of Boston University School of Medicine for manuscript editing, all members of the Kotton laboratory and Alan Fine for scientific discussions, and Lia Cross and Sarah Ohle for technical assistance. The authors also wish to thank Camille Kotton and Kimberly Fisher for constructive criticism.

Received for publication July 3, 2008, and accepted in revised form October 28, 2009.

Address correspondence to: Darrell N. Kotton, The Pulmonary Center, Boston University School of Medicine, 715 Albany St., R-304, Boston, Massachusetts 02118, USA. Phone: (617) 638-4860; Fax: (617) 536-8093; E-mail: dkotton@bu.edu.
1. Yang Y, et al. Cellular and humoral immune responses to viral antigens create barriers to lungdirected gene therapy with recombinant adenoviruses. J Virol. 1995;69(4):2004-2015.

2. Zabner J, et al. Adenovirus-mediated gene transfer transiently corrects the chloride transport defect in nasal epithelia of patients with cystic fibrosis. Cell. 1993;75(2):207-216

3. Grubb BR, et al. Inefficient gene transfer by adenovirus vector to cystic fibrosis airway epithelia of mice and humans. Nature. 1994;371(6500):802-806

4. Young LS, Searle PF, Onion D, Mautner V. Viral gene therapy strategies: from basic science to clinical application. J Pathol. 2006;208(2):299-318.
5. Conrad C, et al. Genetically engineered stem cells for therapeutic gene delivery. Curr Gene Ther. 2007;7(4):249-260.

6. Pan H, Mostoslavsky G, Eruslanov E, Kotton DN, Kramnik I. Dual-promoter lentiviral system allows inducible expression of noxious proteins in macrophages. JImmunol Methods. 2008;329(1-2):31-44.

7. Stecenko AA, Brigham KL. Gene therapy progress and prospects: alpha-1 antitrypsin. Gene Ther. 2003;10(2):95-99.

8. Gregory RJ, et al. Expression and characterization of the cystic fibrosis transmembrane conductance regulator. Nature. 1990;347(6291):382-386.

9. Garver RI Jr, Chytil A, Courtney M, Crystal RG.
Clonal gene therapy: transplanted mouse fibroblast clones express human alpha 1-antitrypsin gene in vivo. Science. 1987;237(4816):762-764.

10. Yang $Y$, et al. Cellular immunity to viral antigens limits E1-deleted adenoviruses for gene therapy. Proc Natl Acad Sci U S A. 1994;91(10):4407-4411.

11. Murphy J, Summer R, Wilson AA, Kotton DN, Fine A. The prolonged life-span of alveolar macrophages. Am J Respir Cell Mol Biol. 2008;38(4):380-385.

12. Murphy GJ, Mostoslavsky G, Kotton DN, Mulligan RC. Exogenous control of mammalian gene expression via modulation of translational termination. Nat Med. 2006;12(9):1093-1099.

13. Sinn PL, et al. Lentivirus vectors pseudotyped with 
filoviral envelope glycoproteins transduce airway epithelia from the apical surface independently of folate receptor alpha. J Virol. 2003;77(10):5902-5910.

14. Wang G, Sinn PL, McCray PB Jr. Development of retroviral vectors for gene transfer to airway epithelia. Curr Opin Mol Ther. 2000;2(5):497-506

15. Kobinger GP, Weiner DJ, Yu QC, Wilson JM. Filovirus-pseudotyped lentiviral vector can efficiently and stably transduce airway epithelia in vivo. Nat Biotechnol. 2001;19(3):225-230.

16. Johnson LG, Olsen JC, Naldini L, Boucher RC. Pseudotyped human lentiviral vector-mediated gene transfer to airway epithelia in vivo. Gene Ther. 2000;7(7):568-574.

17. Landsman L, Jung S. Lung macrophages serve as obligatory intermediate between blood monocytes and alveolar macrophages. J Immunol. 2007;179(6):3488-3494.

18. Maus U, et al. Monocytes recruited into the alveolar air space of mice show a monocytic phenotype but upregulate CD14. Am J Physiol Lung Cell Mol Physiol. 2001;280(1):L58-L68.

19. Kotton DN, Summer RS, Sun X, Ma BY, Fine A. Stem cell antigen-1 expression in the pulmonary vascular endothelium. Am J Physiol Lung Cell Mol Physiol. 2003;284(6):L990-L996.

20. Wagers AJ, Sherwood RI, Christensen JL, Weissman IL. Little evidence for developmental plasticity of adult hematopoietic stem cells. Science. 2002;297(5590):2256-2259

21. Kotton DN, Fabian AJ, Mulligan RC. Failure of bone marrow to reconstitute lung epithelium. Am J Respir Cell Mol Biol. 2005;33(4):328-334.

22. van Oud Alblas AB, van Furth R. Origin, kinetics, and characteristics of pulmonary macrophages in the normal steady state. J Exp Med. 1979;149(6):1504-1518.

23. Jennings JH, Linderman DJ, Hu B, Sonstein J, Curtis $\mathrm{JL}$. Monocytes recruited to the lungs of mice during immune inflammation ingest apoptotic cells poorly. Am J Respir Cell Mol Biol. 2005;32(2):108-117.

24. Maus U, et al. Molecular pathways of monocyte emigration into the alveolar air space of intact mice. Am J Respir Crit Care Med. 2002;165(1):95-100.

25. Wilson AA, et al. Sustained expression of alpha1antitrypsin after transplantation of manipulated hematopoietic stem cells. Am J Respir Cell Mol Biol. 2008;39(2):133-141.

26. American Thoracic Society and European Respiratory Society. American Thoracic Society/European Respiratory Society statement: standards for the diagnosis and management of individuals with alpha-1 antitrypsin deficiency. Am J Respir Crit Care Med. 2003;168(7):818-900.

27. Ito $S$, et al. Tissue heterogeneity in the mouse lung: effects of elastase treatment. J Appl Physiol. 2004;97(1):204-212.

28. Parameswaran $\mathrm{H}$, Majumdar A, Ito S, Alencar AM, Suki B. Quantitative characterization of airspace enlargement in emphysema. J Appl Physiol. 2006;100(1):186-193.

29. Lucey EC, Keane J, Kuang PP, Snider GL, Goldstein $\mathrm{RH}$. Severity of elastase-induced emphysema is decreased in tumor necrosis factor-alpha and interleukin-1beta receptor-deficient mice. Lab Invest.
2002;82(1):79-85.

30. Houghton AM, et al. Elastin fragments drive disease progression in a murine model of emphysema. J Clin Invest. 2006;116(3):753-759.

31. Churg A, et al. Acute cigarette smoke-induced connective tissue breakdown requires both neutrophils and macrophage metalloelastase in mice. $A m$ J Respir Cell Mol Biol. 2002;27(3):368-374.

32. Hashimoto D, et al. Intratracheal administration of third-generation lentivirus vector encoding MPT51 from Mycobacterium tuberculosis induces specific CD8+ T-cell responses in the lung. Vaccine. 2008;26(40):5095-5100

33. Shapiro SD. The macrophage in chronic obstructive pulmonary disease. Am J Respir Crit Care Med. 1999;160(5 pt 2):S29-S32

34. Song $S$, et al. Sustained secretion of human alpha1-antitrypsin from murine muscle transduced with adeno-associated virus vectors. Proc Natl Acad SciUS A. 1998;95(24):14384-14388.

35. Song S, et al. Stable therapeutic serum levels of human alpha-1 antitrypsin (AAT) after portal vein injection of recombinant adeno-associated virus (rAAV) vectors. Gene Ther. 2001;8(17):1299-1306.

36. Brantly ML, et al. Phase I trial of intramuscular injection of a recombinant adeno-associated virus serotype 2 alphal-antitrypsin (AAT) vector in AAT-deficient adults. Hum Gene Ther. 2006;17(12):1177-1186.

37. Brigham KL, et al. Transfection of nasal mucosa with a normal alpha1-antitrypsin gene in alpha1-antitrypsin-deficient subjects: comparison with protein therapy. Hum Gene Ther. 2000;11(7):1023-1032.

38. Takahara H, Nakamura Y, Yamamoto K, Sinohara $\mathrm{H}$. Comparative studies on the serum levels of alpha-1-antitrypsin and alpha-macroglobulin in several mammals. Tohoku J Exp Med. 1983;139(3):265-270.

39. Kushi A, et al. Disruption of the murine alpha1-antitrypsin/PI2 gene. Exp Anim. 2004;53(5):437-443.

40. Wewers MD, et al. Replacement therapy for alpha 1-antitrypsin deficiency associated with emphysema. N Engl J Med. 1987;316(17):1055-1062.

41. Seersholm N, et al. Does alpha1-antitrypsin augmentation therapy slow the annual decline in FEV1 in patients with severe hereditary alpha1-antitrypsin deficiency? Wissenschaftliche Arbeitsgemeinschaft zur Therapie von Lungenerkrankungen (WATL) alpha1-AT study group. Eur Respir J. 1997;10(10):2260-2263.

42. Gottlieb DJ, et al. Short-term supplementation therapy does not affect elastin degradation in severe alpha(1)-antitrypsin deficiency. The American-Italian AATD Study Group. Am J Respir Crit Care Med. 2000;162(6):2069-2072.

43. Churg A, Wang RD, Xie C, Wright JL. alpha-1Antitrypsin ameliorates cigarette smoke-induced emphysema in the mouse. Am J Respir Crit Care Med. 2003;168(2):199-207.

44. Churg A, et al. Alpha-1-antitrypsin and a broad spectrum metalloprotease inhibitor, RS113456, have similar acute anti-inflammatory effects. $L a b$ Invest. 2001;81(8):1119-1131.

45. Petrache I, et al. A novel antiapoptotic role for alpha1-antitrypsin in the prevention of pul- monary emphysema. Am J Respir Crit Care Med. 2006;173(11):1222-1228

46. Shapiro SD. Transgenic and gene-targeted mice as models for chronic obstructive pulmonary disease. Eur Respir J. 2007;29(2):375-378.

47. Godleski JJ, Brain JD. The origin of alveolar macrophages in mouse radiation chimeras. J Exp Med. 1972;136(3):630-643.

48. Matute-Bello G, et al. Optimal timing to repopulation of resident alveolar macrophages with donor cells following total body irradiation and bone marrow transplantation in mice. J Immunol Methods. 2004;292(1-2):25-34

49. Borok Z, et al. Vesicular stomatits virus G-pseudotyped lentivirus vectors mediate efficient apical transduction of polarized quiescent primary alveolar epithelial cells. J Virol. 2001;75(23):11747-11754.

50. Wunderlich S, et al. Type II pneumocyte-restricted green fluorescent protein expression after lentiviral transduction of lung epithelial cells. Hum Gene Ther. 2008;19(1):39-52.

51. Yu ZY, et al. Lentivirus vector-mediated gene transfer to the developing bronchiolar airway epithelium in the fetal lamb. J Gene Med. 2007;9(6):429-439.

52. Buckley SM, et al. Lentiviral transduction of the murine lung provides efficient pseudotype and developmental stage-dependent cell-specific transgene expression. Gene Ther. 2008;15(16):1167-1175.

53. Hendrickson B, et al. Development of lentiviral vectors with regulated respiratory epithelial expression in vivo. Am J Respir Cell Mol Biol. 2007;37(4):414-423.

54. Medina MF, et al. Lentiviral vectors pseudotyped with minimal filovirus envelopes increased gene transfer in murine lung. Mol Ther. 2003;8(5):777-789.

55. Naldini L, et al. In vivo gene delivery and stable transduction of nondividing cells by a lentiviral vector. Science. 1996;272(5259):263-267.

56. Zufferey R, Donello JE, Trono D, Hope TJ. Woodchuck hepatitis virus posttranscriptional regulatory element enhances expression of transgenes delivered by retroviral vectors. J Virol. 1999;73(4):2886-2892.

57. Rennard SI, et al. Estimation of volume of epithelial lining fluid recovered by lavage using urea as marker of dilution. J Appl Physiol. 1986;60(2):532-538.

58. Amar S, Zhou Q, Shaik-Dasthagirisaheb Y, Leeman S. Diet-induced obesity in mice causes changes in immune responses and bone loss manifested by bacterial challenge. Proc Natl Acad Sci U S A. 2007;104(51):20466-20471.

59. Hovav $\mathrm{AH}$, et al. Duration of antigen expression in vivo following DNA immunization modifies the magnitude, contraction, and secondary responses of CD8+ T lymphocytes. J Immunol. 2007;179(10):6725-6733.

60. Ito $S$, et al. Early emphysema in the tight skin and pallid mice: roles of microfibril-associated glycoproteins, collagen, and mechanical forces. Am J Respir Cell Mol Biol. 2006;34(6):688-694.

61. Hautamake RD, Kobayashi DK, Senior RM, Shapiro SD. Requirement for macrophage elastase for cigarette smoke-induced emphysema in mice. Science. 1997;277(5334):2002-2004. 